\title{
Primer reporte de la mutación F1534C asociada con resistencia cruzada a DDT y piretroides en Aedes aegypti en Colombia
}

\author{
María Claudia Atencia ${ }^{1}$, María de Jesús Pérez ${ }^{1}$, María Cristina Jaramillo¹, Sandy Milena Caldera1, \\ Suljey Cochero², Eduar Elías Bejarano \\ 1 Grupo de Investigaciones Biomédicas, Universidad de Sucre, Sincelejo, Colombia \\ 2 Secretaría de Salud de Sucre, Sincelejo, Colombia
}

Introducción. La principal estrategia para el control de Aedes aegypti, vector de los virus del dengue, del chikungunya y del zika, se basa en la utilización de insecticidas con el fin de disminuir su población. Sin embargo, su uso ha implicado que el insecto desarrolle resistencia a estos agentes químicos.

Objetivo. Determinar la presencia de la mutación F1534C asociada con resistencia cruzada al DDT y los piretroides en mosquitos de la especie $A$. aegypti en Sincelejo, Colombia.

Materiales y métodos. El estudio se desarrolló con nueve ejemplares de $A$. aegypti que mostraron resistencia a lambdacialotrina en bioensayos desarrollados por la Secretaría de Salud de Sucre. Se utilizó una reacción en cadena de la polimerasa (PCR) semianidada siguiendo la metodología descrita por Harris, et al., para amplificar el exón 31 del gen para del canal de sodio dependiente de voltaje de $A$. aegypti. Los productos de la PCR se secuenciaron, editaron y analizaron con el programa MEGA 5.

Resultados. En todos los mosquitos evaluados se detectó la presencia del alelo silvestre y mutante del exón 31. En la secuencia de nucleótidos del alelo mutante, se observó la sustitución de timina por guanina, la cual produce el cambio del codón UUC por UGC y conlleva el reemplazo del aminoácido fenilalanina por cisteína en el residuo 1534 de la proteína.

Conclusión. Los nueve mosquitos analizados presentaron un genotipo heterocigoto para la mutación F1534C, cuyo efecto fenotípico es la resistencia al "derribo" (knock-down resistance, kdr) con DDT y piretroides.

Palabras clave: Aedes, mutación, resistencia a los insecticidas, genotipo, control de mosquitos, Colombia. doi: http://dx.doi.org/10.7705/biomedica.v36i3.2834

\section{First report of the F1534C mutation associated with cross-resistance to DDT and pyrethroids in Aedes aegypti from Colombia}

Introduction: The main strategy for the control of Aedes aegypti, vector of dengue, chikungunya and Zika viruses, is based on the use of insecticides to reduce its populations. However, their use has led to insect resistance to these chemicals.

Objective: To determine the presence of the F1534C mutation associated with cross-resistance to DDT and pyrethroids in $A$. aegypti in Sincelejo, Colombia.

Materials and methods: We studied nine specimens of $A$. aegypti that showed resistance to lambdacyhalothrin in bioassays developed by the Secretaría de Salud de Sucre. We used a seminested PCR as previously described by Harris, et al., to amplify exon 31 of the para gene of the voltagedependent sodium channel of $A$. aegypti. We sequenced, edited, and analyzed PCR products with the MEGA 5 software.

Results: We detected the wild and mutant alleles of exon 31 in all of the nine mosquitoes tested, and observed the substitution of thymine for guanine in the nucleotide sequence of the mutant allele, producing a change to UGC in the UUC codon, which led to the replacement of phenylalanine by cysteine in residue 1534 of the protein.

Conclusion: The nine mosquitoes analyzed presented a heterozygote genotype for the F1534C mutation, whose phenotypic effect is knockdown resistance (kdr) to DDT and pyrethroids.

Key words: Aedes, mutation, insecticide resistance, genotype, mosquito control, Colombia. doi: http://dx.doi.org/10.7705/biomedica.v36i3.2834

\footnotetext{
Contribución de los autores:

María Claudia Atencia: procesamiento de muestras, análisis molecular y redacción del manuscrito

María de Jesús Pérez: procesamiento de muestras y análisis molecular

María Cristina Jaramillo y Sandy Milena Caldera: diseño metodológico, asesoría en los ensayos y redacción del manuscrito

Suljey Cochero: recolección de material biológico y bioensayos

Eduar Elías Bejarano: orientación para el análisis de los datos y redacción del manuscrito
} 
El mosquito Aedes aegypti (Linnaeus, 1762) (Diptera: Culicidae) es el principal vector de las arbovirosis de mayor importancia en salud pública, como la fiebre amarilla urbana, el dengue (1), el chikungunya (2) y el zika. El dengue se considera una enfermedad reemergente en países tropicales; se estima que 390 millones de casos ocurren anualmente y que más del $55 \%$ de la población mundial se encuentra en riesgo de transmisión en 128 países donde habitan 824 millones de personas (3). Desde finales de los ochenta, el dengue es considerado endémico en Colombia debido a los altos índices de infestación con $A$. aegypti y a su distribución en el $90 \%$ del territorio nacional (4). La estrategia para prevenir o reducir la transmisión de dengue en el mundo se fundamenta, principalmente, en el control del vector $A$. aegypti mediante el uso de insecticidas para disminuir y erradicar las poblaciones del mosquito (5) e interrumpir el contacto entre el humano y el vector (6). Además, se están empleando nuevas estrategias orientadas a la modificación del insecto mediante técnicas de ingeniería genética y biotecnología para reducir su población (7).

Las principales clases de insecticidas utilizados actualmente para el control vectorial son los organofosforados, los carbamatos y los piretroides (8). El uso intensivo y extensivo de estos agentes químicos indujo la aparición de resistencia a los insecticidas en insectos de importancia médica (9). Aedes aegypti ha desarrollado resistencia a una amplia variedad de insecticidas (9), lo que constituye uno de los principales obstáculos para su control. El mosquito ha adquirido resistencia especialmente a insecticidas del tipo de los piretroides, uno de los grupos químicos más utilizados desde los años noventa para el control vectorial (10). Esta resistencia puede estar asociada con mecanismos metabólicos, como las enzimas de desintoxicación, o con mutaciones no sinónimas en el gen para, el cual codifica para el canal de sodio dependiente del voltaje de las neuronas de los mosquitos. Se han reportado varias mutaciones en este canal, pero solamente unas cuantas se han asociado claramente con la resistencia a los insecticidas, al comprobar que producen insensibilidad en el sitio de anclaje del insecticida al canal: V1016I,

\footnotetext{
Correspondencia:

María Claudia Atencia, Universidad de Sucre, Carrera $14 \mathrm{~N}^{\circ}$ 16B-32, Sincelejo, Colombia

Teléfono: (575) 282 0830; fax: (575) 2821240

mariclau_1112@hotmail.com

Recibido: 18/05/15; aceptado: 20/03/16
}

asociada con la resistencia a piretroides de los tipos I y II, así como al DDT; V1016G, asociada con la resistencia a la deltametrina, la permetrina y al DDT, y la F1534C, asociada con la resistencia a la permetrina y el DDT $(11,12)$. El efecto fenotípico de estas mutaciones es la resistencia al "derribo" o kdr (knock-down resistance), mecanismo que confiere resistencia cruzada entre los insecticidas piretroides y el DDT (12).

En Colombia, las poblaciones de $A$. aegypti han sido muy presionadas por el uso de distintos tipos de insecticidas (13) (Salazar M, Carvajal A, Cuéllar M, Olaya A, Quiñones J, Velásquez O, et al. Resistencia a insecticidas en poblaciones de Aedes aegypti y Anopheles spp. en los departamentos de Huila, Valle, Cauca y Nariño. Memorias, XIII Congreso Colombiano de Parasitología y Medicina Tropical. Biomédica. 2007;27(Supl.2):177). Estudios sobre el estado actual de la resistencia en las poblaciones del mosquito en el país indican que existe resistencia al DDT y disminución de la sensibilidad al larvicida temefós, al malatión y a la lambdacialotrina (Santacoloma L, Brochero HL, Chávez B. Estado de la susceptibilidad (sic) a insecticidas en Aedes aegypti (Linnaus 1762) (Diptera: Culicidae) en cinco departamentos de Colombia. Memorias, XIII Congreso Colombiano de Parasitología y Medicina Tropical. Biomédica. 2007;27(Supl.2):175). En el 2008 se detectó en Sincelejo, Sucre, un alto nivel de resistencia al larvicida temefós y al DDT, se confirmó la sensibilidad al malatión y el fenitrotión, y se encontró una disminución de la sensibilidad a piretroides en poblaciones del mosquito $A$. aegypti de las localidades de El Cortijo y Botero (14). En el 2014, Maestre, et al. (15), reportaron en la población de $A$. aegypti de Sincelejo poca resistencia a la permetrina y el ciflutrín, resistencia moderada a la deltametrina y el metil-pirimifós y alta resistencia a la lambdacialotrina y el DDT. En este estudio también se confirmó la sensibilidad al malatión y el fenitrotión.

Actualmente, el malatión es el insecticida utilizado para el control de $A$. aegypti en el municipio de Sincelejo, ya que hay pocos reportes de resistencia del mosquito a este agente químico en el país. Solo en el departamento de Antioquia se ha informado sobre la resistencia local a dicho insecticida (16), pero no en otras partes del país, aunque en un estudio reciente se observó una disminución en la sensibilidad al malatión en las localidades de Campoalegre (Huila) y Palmira (Valle del Cauca) (17). Los piretroides, como la lambdacialotrina y la deltametrina, se dejaron de utilizar para el control 
vectorial en Sucre, porque se encontró poca sensibilidad a ellos (Secretaría de Salud de Sucre, datos sin publicar).

En este contexto, el objetivo del presente trabajo fue evaluar la presencia de la mutación F1534C asociada con resistencia cruzada a DDT y piretroides en mosquitos de la especie $A$. aegypti en Sincelejo, Colombia.

\section{Materiales y métodos}

El estudio se hizo con nueve mosquitos de $A$. aegypti suministrados por la Secretaría de Salud de Sucre, pertenecientes a la generación F2 de individuos capturados en estado larvario en las localidades de Puerta Roja y Las Delicias, área urbana de Sincelejo, capital del departamento de Sucre. En estos mosquitos se había observado resistencia a la lambdacialotrina en bioensayos de sensibilidad, con porcentajes de mortalidad que alcanzaron el $77,02 \%$ en la localidad de Puerta Roja y el 32,63 \% en Las Delicias. Los bioensayos se desarrollaron según la metodología de la Organización Mundial de la Salud (18) como parte de las actividades rutinarias de vigilancia de vectores de la Secretaría de Salud de Sucre. Se hicieron tres réplicas con 20 individuos para la exposición al insecticida; cada repetición incluyó cuatro tratamientos y un control. Con base en la evidencia bioquímica de resistencia de estos mosquitos al insecticida lambdacialotrina (Secretaría de Salud de Sucre, datos sin publicar), se evaluó molecularmente la presencia de una de las mutaciones kdr que confiere resistencia a insecticidas.

Para el estudio molecular, se extrajo el ADN total de cada uno de los nueve mosquitos según el protocolo descrito por Caldera, et al. (19), pero modificando el tiempo de incubación con proteinasa $\mathrm{K}$, el cual se ajustó a cinco horas, a una temperatura de $65^{\circ} \mathrm{C}$. La amplificación del exón 31 del gen para, que codifica para el segmento transmembrana 6 del dominio III (dominio IIIS6) del canal de sodio dependiente de voltaje en $A$. aegypti, se hizo mediante reacción en cadena de la polimerasa (PCR) semianidada, utilizando la tétrada de cebadores descrita por Harris, et al. (20): AaEx31P (5'-TCGCGGGAGGTAAGTTATTG-3'), AaEx31Q (5'-GTTGATGTGCGATGGAAATG-3'), AaEx31wt (5'-CCTCTACTTTGTGTTCTTCATCATCTT-3') y AaEx31mut (5'-GCGTGAAGAACGACCCGC-3').

La primera fase consistió en la amplificación de una banda de $350 \mathrm{pb}$, que incluyó todo el exón 31, con los cebadores $\mathrm{AaEx31P}$ y $\mathrm{AaEx31Q}$ en un volumen final de $25 \mu$ que contenían 2,5 mM de $\mathrm{MgCL}_{2}, 0,3$ $\mathrm{mM}$ de dNTP, $0,5 \mu \mathrm{M}$ de solución tampón de PCR 1X, 2,5 unidades de Taq polimerasa y $0,5 \mu \mathrm{l}$ de la solución con el ADN, y se completó con agua ultrapura estéril. En la segunda fase se usaron los cebadores AaEx31wt y AaEx31Q para amplificar una banda de $231 \mathrm{pb}$, la cual correspondía al alelo silvestre que codifica para fenilalanina en el residuo 1534 de la proteína, y los cebadores AaEx31P y AaEx31mut para la amplificación de una banda de 163 pb, la cual caracteriza al alelo mutante que codifica para cisteína en el mismo residuo. Cada reacción se hizo por separado bajo las condiciones de amplificación descritas. En cada serie de PCR se incluyó un control negativo constituido por agua estéril en lugar de ADN, con el fin de establecer si existía contaminación. El perfil térmico consistió en una etapa inicial de desnaturalización a $95^{\circ} \mathrm{C}$ durante cinco minutos, seguida por 35 ciclos de desnaturalización a $94{ }^{\circ} \mathrm{C}$ durante 30 segundos, alineamiento a $63^{\circ} \mathrm{C}$ durante 30 segundos, extensión a $72{ }^{\circ} \mathrm{C}$ durante 30 segundos, y una etapa final de extensión a $72{ }^{\circ} \mathrm{C}$ durante 10 minutos.

Los productos de PCR se separaron por electroforesis en gel de agarosa al $2 \%$ (peso/volumen), desarrollada en solución tampón TBE 0,5X a 100 $\checkmark$ durante 40 minutos. El ADN se tiñó con GelStar y se visualizó con luz ultravioleta. Para establecer el tamaño de las bandas, se incluyó un marcador de peso molecular de 100 pb HyperLadder V. Los productos amplificados se secuenciaron mediante electroforesis capilar en un secuenciador automático, usando los mismos cebadores empleados para la PCR. Las secuencias obtenidas a partir de los electroforegramas resultantes de la secuenciación se editaron y alinearon con el programa MEGA, versión 5.0 (21). Con cada secuencia consenso obtenida se hizo una búsqueda en BLASTN (22) para establecer con cuáles de las secuencias disponibles en GenBank (http://www.ncbi.nlm.nih.gov/genbank) presentaban la mayor similitud. Las secuencias de nucleótidos se tradujeron a aminoácidos con base en el código genético estándar. Todas las secuencias obtenidas durante esta investigación se depositaron en GenBank, con los números de acceso KP861896, KP861897 y KP861898.

\section{Resultados}

En la primera fase de la PCR anidada en los nueve especímenes de $A$. aegypti de Sincelejo se amplificó una banda de 350 pb del exón 31 del gen para, el cual codifica para el dominio IIIS6 del canal de sodio dependiente de voltaje (figura 1A). 
Además, durante la segunda fase de amplificación en cada uno de los mosquitos se obtuvo una banda de $231 \mathrm{pb}$ del alelo silvestre y una de $163 \mathrm{pb}$ del alelo mutante (figura 1B). La secuenciación de los 27 amplicones totales generó nueve secuencias de nucleótidos consenso de 325 bases (número de

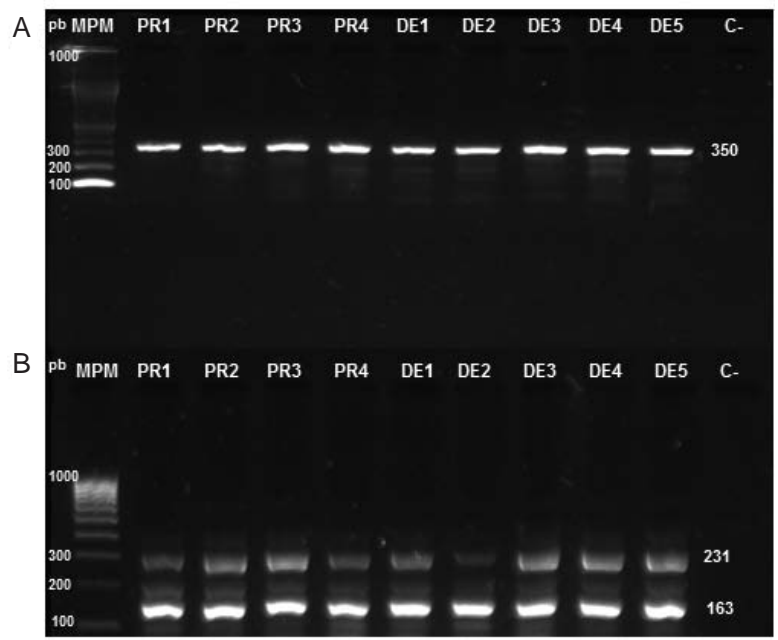

Figura 1. Electroforesis en gel de agarosa al $2 \%$ de los productos de la PCR del exón 31 del gen para, el cual codifica para el dominio IIIS6 del canal de sodio dependiente de voltaje. MPM: marcador de peso molecular de 100 pb HyperLadder V; carriles PR1 a DE5: productos amplificados en nueve individuos de Aedes aegypti de las localidades de Puerta Roja (PR) y Las Delicias (DE), Sincelejo, Colombia; carril C-: control negativo. A. Banda de 350 pb correspondiente al exón 31 completo. B. Banda de 231 pb del alelo silvestre y de 163 $\mathrm{pb}$ del alelo mutante acceso: KP861896) correspondientes al exón 31 completo del gen para, nueve secuencias de 152 nucleótidos del alelo mutante (número de acceso: KP861897) y nueve secuencias de 216 nucleótidos del alelo silvestre (número de acceso: KP861898).

El alineamiento de las tres secuencias (exón completo, alelo mutante y alelo silvestre) de cada individuo permitió identificar una región de 31 nucleótidos, la cual fue común a todas las secuencias e iba desde la posición 42 hasta la 72 (figura 2). En la posición 55 del alineamiento se evidenció un cambio de timina $(T)$ a guanina $(G)$ en los nueve individuos analizados, de tal manera que, mientras en la secuencia del alelo silvestre se observó una $\mathrm{T}$, en la secuencia del exón 31 completo y del alelo mutante se presentó una $\mathrm{G}$.

La comparación de las secuencias de los nueve mosquitos de Sincelejo, con una secuencia homóloga de $A$. aegyptisensible a insecticidas (aislamiento 1534Phe, número de acceso KF527415.1) y una resistente a permetrina (aislamiento 1534Cys, número de acceso KF527414.1), puso en evidencia que el cambio de $T$ por G (figura 2) afectaba específicamente el codón UUC, el cual cambió a UGC y codificó para el residuo 1534 del dominio IIIS6 del canal de sodio dependiente de voltaje, al igual que en la secuencia homóloga de referencia del también díptero Musca domestica (número de acceso: NM 001286885) (23). Esta sustitución no sinónima condujo al cambio de fenilalanina por cisteína en la proteína.

111111111122222222223333333333444444444455555555556666666666777 1234567890123456789012345678901234567890123456789012345678901234567890123456789012 KF527415.1 GGAAAGCAGCCGATTCGCGAGACCAACATCTACATGTACCTCTACTTTGTGTTCTTCATCATCTTCGGGTCGTTCTTCACGC KP861898.1 G. KP861898.1

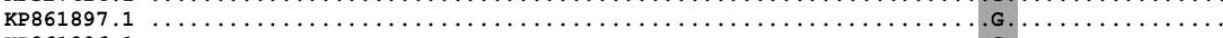

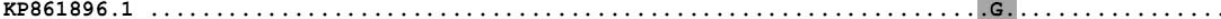

111111111111111111111111111111111111111111111111111111 7777777888888888899999999990000000000111111111122222222223333333333444444444455555 3456789012345678901234567890123456789012345678901234567890123456789012345678901234 3456789012345678901234567890123456789012345678901234567890123456789012345678901234
KF527415.1 TGAATCTGTTCATCGGTTCATCATCGACAACTTCAACGAGCAGAAGAAGAAAGCCGGGGCTCACTGGAAATTTCATGAC

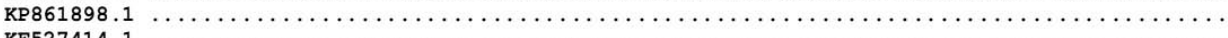

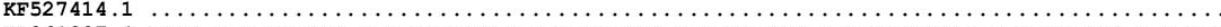
KP861897.1 - 1 (-

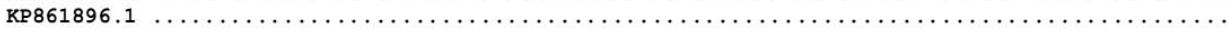

111111111111111111111111111111111111111111111222222222222222222222222222222222222 55555666666666677777777778888888888999999999900000000001111111112222222222333333 567890123456789012345678901234567890123456789012345678901234567890123456789012345 KF527415.1 GGAGGATCAGAAAAAGTACTACAACGCCATGAAAAAGATGGGCTCGAAGAAGCCGCTGAAAGCTATTCCACGGCCTAGGGT

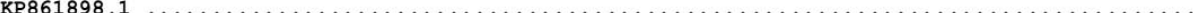

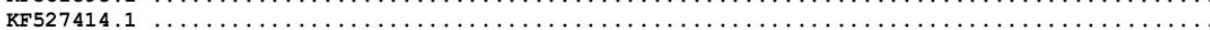
KF527414.1 KP861896.1

Figura 2. Alineamiento de las secuencias de nucleótidos del exón 31 del gen para, el cual codifica para el dominio IIIS6 del canal de sodio dependiente de voltaje de Aedes aegypti. Los números de acceso corresponden a las secuencias del alelo silvestre (número de acceso: KP861898.1), las secuencias del alelo mutante (número de acceso: KP861897.1), las secuencias derivadas de la banda de control (número de acceso: KP861896.1), la secuencia de un alelo silvestre de Ae. aegypti tomada de GenBank (número de acceso: KF527415.1) y la secuencia de un alelo mutante del mosquito también tomada de GenBank (número de acceso: KF527414.1). Los puntos denotan homología y, los guiones, ausencia de información. 


\section{Discusión}

La presencia de los alelos silvestre y mutante en los nueve individuos de $A$. aegypti de las localidades de Las Delicias y Puerta Roja (Sincelejo) indica que estos mosquitos son heterocigotos para la mutación F1534C, la cual consiste en el cambio de fenilalanina por cisteína en la proteína y confiere resistencia cruzada a los piretroides, el DDT y sus análogos (12). Este hallazgo constituye el primer reporte de la mutación $\mathrm{F} 1534 \mathrm{C}$ en $A$. aegypti de Colombia.

En estudios previos del vector en Gran Caimán (20), México (24), Brasil (25), India (26) y Venezuela (27), la mutación F1534C se encontró asociada con resistencia al piretroide permetrina y al organoclorado DDT. Asimismo, en una investigación desarrollada en poblaciones de $A$. aegypti de Tailandia (28), se halló una mutación que afectaba el residuo 1552 del dominio IIIS6 del canal de sodio dependiente de voltaje, consistente en el cambio de $T$ por $\mathrm{G}$ en la segunda posición del codón, el cual produce la sustitución de fenilalanina por cisteína en la proteína y confiere resistencia a piretroides de tipo II.

En lo que respecta a Colombia, en poblaciones de A. aegypti de los municipios de Puerto Colombia, Soledad, Valledupar, San Juan del Cesar, Sincelejo, Montería y Ciénaga, y los distritos de Barranquilla y Cartagena en la región Caribe, se encontró la mutación kdr Val1016lle, la cual se ha relacionado con resistencia a los piretroides deltametrina, ciflutrín y permetrina, pero no a lambdacialotrina (15), en mosquitos que portaban la mutación en condición homocigota.

A pesar del limitado número de ejemplares procesados y de la evaluación de una sola mutación, lo cual obedeció al carácter exploratorio del presente estudio, los resultados de esta investigación podrían explicar la resistencia a la lambdacialotrina observada en los bioensayos con mosquitos de las localidades de Puerta Roja y Las Delicias de Sincelejo, sometidas a control químico con lambdacialotrina y deltametrina, lo cual se suma a reportes previos de resistencia a piretroides en el 2012 (4) y el 2013 (Secretaría de Salud de Sucre, datos sin publicar). Más aún, estos resultados coinciden con los estudios de Anaya (14), quien encontró un alto nivel de resistencia a DDT en mosquitos de las localidades de El Cortijo y Botero en la misma ciudad, así como una disminución de la sensibilidad a los piretroides lambdacialotrina y deltametrina.
Teniendo en cuenta que la mutación F1534C confiere resistencia cruzada a piretroides y DDT, es de esperar que los mosquitos de Las Delicias y Puerta Roja también presenten resistencia al DDT. De allí la importancia de que las entidades de salud estatales, con el apoyo de los centros de investigación, ejerzan una vigilancia continua de la sensibilidad de las poblaciones de $A$. aegypti a los insecticidas en esta ciudad, y que se tengan en cuenta los resultados del presente estudio a la hora de tomar decisiones concernientes al control químico del vector.

\section{Agradecimientos}

A la Secretaría de Salud de Sucre por proporcionar los mosquitos usados en el estudio.

\section{Conflicto de intereses}

Los autores declaramos que no hubo conflictos de intereses.

\section{Financiación}

Este trabajo se desarrolló en el marco del proyecto de investigación "Determinación de la variabilidad genética entre poblaciones de Aedes (Stegomyia) aegypti (Diptera: Culicidae), vector del virus del dengue en Colombia (Código 112951929257)", financiado por el Departamento Administrativo de Ciencia, Tecnología e Innovación, Colciencias.

\section{Referencias}

1. Trujillo $\mathbf{M}$, Marquetti $\mathbf{M}$, Vásquez $\mathbf{A}$, Montes J. Dinámica estacional y temporal de Aedes aegypti (Diptera: Culicidae) en el municipio Cienfuegos. Rev Cubana Med Trop. 2010;62:98-106.

2. World Health Organization. Guidelines for prevention and control of chikungunya fever. World Health Organization, Regional Office for South-East Asia, New Delhi: WHO; 2009. Fecha de consulta: 12 de septiembre de 2014. Disponible en: http://www.wpro.who.int/mvp/topics/ntd/Chikungunya_ WHO_SEARO.pdf

3. Bhatt S, Gething PW, Brady OJ, Messina JP, Farlow AW, Moyes CL, et al. The global distribution and burden of dengue. Nature. 2013;496:504-7. http://dx.doi.org/10.1038/ nature12060

4. Instituto Nacional de Salud. Guía de atención clínica integral del paciente con dengue. Fecha de consulta: 20 de diciembre de 2014. Disponible en: http://www2.paho.org/ $\mathrm{col} / \mathrm{dmdocuments/Guiadengue210310.pdf}$

5. Bisset JA, Rodríguez MM, San Martín JL, Romero JE, Montoya R. Evaluación de la resistencia a insecticidas de una cepa de Aedes aegypti de El Salvador. Rev Panam Salud Pública. 2009;26:229-34. http://dx.doi.org/10.1590/ S1020-49892009000900007

6. Morrison AC, Zielinski-Gutiérrez E, Scott TW, Rosenberg R. Defining challenges and proposing solutions for control of the virus vector Aedes aegypti. PLoS Med. 2008;5:e68. http://dx.doi.org/10.1371/journal.pmed.0050068 
7. Achee NL, Gould F, Perkins TA, Reiner RC, Morrison AC, Ritchie SA, et al. A critical assessment of vector control for dengue prevention. PLoS Negl Trop Dis. 2015;9:e0003655. http://dx.doi.org/10.1371/journal.pntd.0003655

8. van Den Berg H, Zaim M, Singh R, Soares A Ameneshewa B, Mnzava A, et al. Global trends in the use of insecticides to control vector-borne diseases. Environ Health Perspect. 2012;20:577-82. http://dx.doi.org/10.1289/ehp.1104340

9. Ranson H, Burhani J, Lumjuan N, Black WC. Insecticide resistance in dengue vectors. TropIKA.net. 2010;1:1-12.

10. Maestre R, Rey G, De Las Salas J, Vergara C, Santacoloma L, Goenaga S, et al. Estado de la susceptibilidad de Aedes aegypti a insecticidas en Atlántico (Colombia). Rev Colomb Entomol. 2010;36:242-8.

11. Dong K, Du Y, Rinkevich F, Nomura Y, Xu P, Wang L, et al. Molecular biology of insect sodium channels and pyrethroid resistance. Insect Biochem Mol Biol. 2014;50:1-17. http:// dx.doi.org/10.1016/j.ibmb.2014.03.012

12. Brengues C, Hawkes NJ, Chandre F, Mccarroll L, Duchon S, Guillet P, et al. Pyrethroid and DDT crossresistance in Aedes aegyptiis correlated with novel mutations in the voltage-gated sodium channel gene. Med Vet Entomol. 2003;13:87-94. http://dx.doi.org/10.1046/j. 1365-2915.2003.00412.x

13. Fonseca I, Quiñones ML. Resistencia a insecticidas en mosquitos (Diptera: Culicidae): mecanismos, detección y vigilancia en salud pública. Rev Colomb Entomol. 2005;31:107-15.

14. Anaya YP. Evaluación de la susceptibilidad a insecticidas en Aedes aegypti capturados en el municipio de Sincelejo, departamento de Sucre, Colombia (tesis). Sincelejo: Universidad de Sucre; 2008.

15. Maestre R, Gómez D, Ponce G, Flores AE. Susceptibility to insecticides and resistance mechanisms in Aedes aegypti from the Colombian Caribbean Region. Pestic Biochem Physiol. 2014;116:63-73. http://dx.doi.org/10.1016/j.pestbp. 2014.09.014

16. Mazzari M. Revisión del estado actual de la resistencia en Aedes aegypti a insecticidas utilizados en salud pública. Bol Mal Salud Amb. 1995;35:90-5.

17. Ocampo CB, Salazar MJ, Mina NJ, Mcallister J, Brogdon W. Insecticide resistance status of Aedes aegypti in 10 localities in Colombia. Acta Trop. 2011;118:37-44. http://dx. doi.org/10.1016/j.actatropica.2011.01.007

18. World Health Organization.Insecticide resistance and vector control: WHO; 1970. Fecha de consulta: 22 de agosto de 2014. Disponible en: http://apps.who.int/iris/ bitstream/10665/40771/1/WHO_TRS_443_(part1).pdf
19. Caldera SM, Jaramillo MC, Cochero S, Pérez A, Bejarano EE. Diferencias genéticas entre poblaciones de Aedes aegypti de municipios del norte de Colombia, con baja y alta incidencia de dengue. Biomédica. 2013;33:89-98. http://dx. doi.org/10.7705/biomedica.v33i0.1573

20. Harris AF, Shavanthi R, Ranson H. Pyrethroid resistance in Aedes aegypti from Grand Cayman. Am J Trop Med Hyg. 2010;83:277-84. http://dx.doi.org/10.4269/ajtmh.2010. 09-0623

21. Tamura K, Peterson D, Peterson N, Stecher G, Nei M, Kumar S. MEGA5: Molecular evolutionary genetics analysis using maximum likelihood, evolutionary distance, and maximum parsimony methods. Mol Biol Evol. 2011;28: 2731-9. http://dx.doi.org/10.1093/molbev/msr121

22. Altschul SF, Gish W, Miller W, Myers EW, Lipman DJ. Blast. Basic local alignment search tool. J Mol Biol. 1990;215:40310. http://dx.doi.org/10.1016/S0022-2836(05)80360-2

23. Williamson MS, Martínez D, Hick CA, Devonshire AL. Identification of mutations in the housefly para-type sodium channel gene associated with knockdown resistance $(k d r)$ to pyrethroid insecticides. Mol Gen Genet. 1996;252:51-60.

24. Aponte HA, Penilla RP, Dzul-Manzanilla F, Che-Mendoza A, López AD, Solís $F$, et al. The pyrethroid resistance status and mechanisms in Aedes aegypti from the Guerrero state, México. Pest Biochem Physiol. 2013;107:226-34. http://dx.doi.org/10.1016/j.pestbp.2013.07.005

25. Birggitt JG, Brito PL, Azambuja G, Saori A, Vieira R, Pereira JB, et al. Distribution and dissemination of the Val1016lle and Phe1534Cys Kdrmutations in Aedes aegypti Brazilian natural populations. Parasit Vectors. 2014;7:25. http://dx.doi.org/10.1186/1756-3305-7-25

26. Kushwah RB, Dykes CL, Kapoor N, Adak T, Singh OP. Pyrethroid-resistance and presence of two knockdown resistance $(k d r)$ mutations, F1534C and a novel mutation T1520I, in Indian Aedes aegypti. PLoS Negl Trop Dis. 2015;91:e3332. http://dx.doi.org/10.1371/journal.pntd. 0003332

27. Álvarez LC, Ponce G, Saavedra-Rodríguez K, López B, Flores AE. Frequency of V1016I and F1534C mutations in the voltage-gated sodium channel gene in Aedes aegypti in Venezuela. Pest Manag Sci. 2015;71:863-9. http://dx.doi. org/10.1002/ps.3846

28. Yanola J, Somboon P, Walton C, Nachaiwieng W, Prapanthadara L. A novel F1552/C1552 point mutation in the Aedes aegypti voltage-gated sodium channel gene associated with permethrin resistance. Pestic Biochem Physiol. 2010;96:127-31. http://dx.doi.org/10.1016/j.pestbp. 2009.10.005 